\title{
Frequent item set mining with TM algorithm and tree creation
}

\author{
G. Ramesh Kumar ${ }^{*}$, K. Arulanandam², A. Kavitha ${ }^{3}$ \\ ${ }^{1}$ PG \& Research, Dept of Computer Science \& Applications, Govt Thirumagal Mills College, Thiruvalluvar University, \\ Vellore, India \\ ${ }^{2}$ PG \& Research Dept of Computer Science and Applications, Govt Thirumagal Mills College, Gudiyatham, India \\ ${ }^{3}$ PG \&Research Department of Computer Science and Applications, DG Vaishnav College, Arumbakkam, Chennai-106, India
}

Corresponding Author Email: grk92804@rediffmail.com

https://doi.org/10.18280/ama_b.610401

Received: 10 September 2017

Accepted: 16 March 2018

\author{
Keywords: \\ Frequent Item, FP-Growth, Support and \\ Confident
}

\begin{abstract}
Item set mining is a skill widely recycled in data mining for determining cherished correlations amongst data. The useful measure to extract the knowledge based on user interest is by means of frequency. This is the mostly used technique in order to get the data based on the user preferences and user request, so I have proposed frequent item set mining for searching key element, here mining top-k frequent closed item sets without minimum support should be more preferable than the traditional minimum support-based mining. The recital and suppleness for mining top-k frequent closed item sets, as well as mining top-k frequent closed item sets in data stream milieus and mining top-k frequent closed chronological or tight patterns. Mining top-k numerous closed item sets of Length no less than $\mathrm{k}$ value it will indiscriminately quarried.

This paper introduces a novel calculation for mining complete continuous item sets. This calculation is alluded to as the TM (Transaction Mapping) calculation from here on. In this calculation, exchange ids of everything set are mapped and compacted to nonstop exchange interims in an alternate space and the numbering of item sets is performed by crossing these interim records in a profundity first request along the lexicographic tree. At the point, when the pressure coefficient winds up noticeably littler than the normal number of comparisons for interims crossing point at a specific level, the calculation changes to exchange and convergence.

The calculation against two prominent continuous things set mining calculations, FPdevelopment, utilizing an assortment of informational indexes with short and long successive examples. Exploratory information demonstrates that the TM calculation outflanks these two calculations.
\end{abstract}

\section{INTRODUCTION}

Item set mining is exceptionally famous information mining method and it discovers connections among the distinctive elements of records (for instance, exchange records [1, 5]). Since the presentation of regular item sets in has gotten a lot of consideration in the field of information revelation and information mining. Visit item set mining prompts the disclosure of affiliations and connections among things in expansive value-based or social informational collections. With huge measures of information persistently being gathered and put away, numerous ventures are getting to be noticeably keen on mining such examples from their databases. The disclosure of fascinating connection connections among colossal measures of business exchange records can help in numerous businesses basic leadership procedures, for example, inventory configuration, cross-promoting, and client shopping conduct investigation.

A normal case of successive thing set mining is market bushel examination. This procedure investigates client purchasing propensities by discovering relationship between the distinctive things that clients put in their "shopping wicker bin". The revelation of these affiliations can help retailers create showcasing techniques by picking up understanding into which things are every now and again bought together by clients. Frequency supports any extracted knowledge and is the most common and perhaps useful measure of user interest. It's definitely the most studied:

During the last decade, many researchers have investigated the computational problem of mining patterns that satisfy a user-defined minimum frequency threshold. The simplest form of frequent pattern [3] is the frequent item set. Assumed a catalogue of trades (a transaction being a set of items) we search for deal separations (item sets) that regularly appear composed. By frequently we unkind a number of periods no less than a given verge, this computational problematic is at the root of the well-known connotation rules excavating.

\subsection{Association rule mining}

Visit designs[4], as the name recommends, are examples that happen every now and again in information, There are numerous sorts of regular examples, including successive item sets, visit subsequences (otherwise called consecutive examples), and continuous substructures. A successive thing set commonly alludes to an arrangement of things that regularly seem together in a value-based informational index for instance, drain and bread, which are as often as possible 
purchased together in supermarkets by numerous clients. An as often as possible happening subsequence, for example, the example that clients, tend to buy initial a portable workstation, trailed by a computerized camera, and afterward a memory card, is a (visit) consecutive example. A substructure can allude to various basic structures (e.g., charts, trees, or cross sections) that might be joined with item sets or subsequences. In the event that a substructure happens every now and again, it is known as a (regular) structure design. Mining incessant examples prompts the revelation of fascinating affiliations and relationships inside information.

\section{LITERATURE SURVEY}

As we have seen, much of the time the Apriori hopeful create and-test technique essentially lessens the extent of applicant sets, prompting great execution pick up. Notwithstanding, it can experience the ill effects of two nontrivial costs.

It may in any case need to produce countless sets. For instance, if there are 104 regular 1-itemsets, the Apriori calculation should create more than 107 applicant 2-itemsets. It may need to over and again filter the entire database and check an extensive arrangement of competitors by example coordinating. It is exorbitant to go over every exchange in the database to decide the support of the competitor item sets.

Association rules are the primary method for information mining [6]. The Apriori calculation is a traditional calculation in mining affiliation rules. With the time various changes proposed in Apriori to improve the execution in term of time and number of database passes. For the two bottlenecks of successive thing sets mining, the huge large number of competitor 2-itemsets, the poor productivity of checking their help. This paper principle center lies in the era of successive examples which is the most critical undertaking in clarification of the essentials of affiliation control mining [6]. This is finished by dissecting the usage of the outstanding affiliation run mining calculations Apriori and Proposed calculation Set operation for Frequent Item utilizing Transaction database.

Visit item set mining [7] has been considered broadly in writing. Most past investigations require the determination of a min_support edge and go for mining an entire arrangement of incessant item sets fulfilling min_support [7]. Notwithstanding, by and by, it is troublesome for clients to give a fitting min_support edge. Furthermore, a total arrangement of successive item sets is substantially less conservative than an arrangement of successive shut item sets. In this paper, they propose an option mining undertaking: mining top-k visit shut item sets of length no not exactly min_l, where $\mathrm{k}$ is the coveted number of incessant shut item sets to be mined, and min_l is the insignificant length of each item set. A proficient calculation, called TFP, is created for mining such item sets without min_support. Beginning at min_support $=0$ and by making utilization of the length requirement and the properties of best $\mathrm{k}$ visit shut item sets, min_support can be raised adequately what's more, FP-Tree can be pruned powerfully both amid and after the development of the tree utilizing our two proposed techniques: the shut hub tally and descendant sum. Besides, mining is further speeded up by utilizing a best down and base up consolidated FP-Tree crossing methodology, an arrangement of inquiry space pruning strategies, a quick 2-level hash-listed outcome tree, and a novel shut item set confirmation conspire. Our broad execution thinks about demonstrates that TFP has elite and straight adaptability as far as the database estimate.

Mining high utility itemsets from a value-based database alludes to the revelation of itemsets with high utility like benefits [8]. In spite of the fact that various important calculations have been proposed as of late, they acquire the issue of creating a substantial number of applicant itemsets for high utility itemsets. Such an expansive number of hopeful itemsets debases the mining execution regarding execution time and space necessity. Test comes about demonstrate that the proposed calculations, particularly UP Growth+, reduce the quantity of applicants viably as well as outflank different calculations generously as far as runtime, particularly when databases contain bunches of long exchanges.

Visit weighted item sets speak to connections often holding in information in which things may weight in an unexpected way [9]. In any case, in a few settings, e.g., when the need is to limit a specific cost work, finding uncommon information relationships is more fascinating than mining incessant ones. This paper handles the issue of finding uncommon and weighted item sets, i.e., the rare weighted item set (IWI) mining issue. Two novel quality measures are proposed to drive the IWI mining process. Besides two calculations that perform IWI and Minimal IWI mining productively, determined by the proposed measures, are displayed. Exploratory outcomes indicate proficiency and adequacy of the proposed approach.

Earlier takes a shot at this issue all utilize a two-stage, hopeful era approach with one special case that is however wasteful and not adaptable with substantial databases[10]. The two-stage approach experiences versatility issue because of the enormous number of applicants. This paper proposes a novel calculation that discovers high utility examples in a solitary stage without producing competitors. The curiosities lie in a high utility example development approach, a look ahead technique, and a direct information structure. Solidly, our example development approach is to look through an invert set list tree and to prune seek space by utility upper bouncing. We additionally look forward to distinguish high utility examples without count by a conclusion property and a singleton property. Our straight information structure empowers us to process a tight headed for intense pruning and to straightforwardly distinguish high utility examples in a productive and versatile way, which focuses on the underlying driver with earlier calculations. Broad examinations on inadequate and thick, manufactured and genuine information propose that our calculation is up to 1 to 3 requests of greatness more productive and is more versatile than the best in class calculations. P. Janarthanan et al. has been analyzed that how to retrieve the relevant items using graph theory approaches. The approaches are using index to identify the items in the corpus and also it is efficient kind of finding relevant information [12].

\section{PROPOSED WORK}

Assume that, as a promoting administrator at AllElectronics, you need to know which things are regularly bought together (i.e., inside a similar exchange). Aincident of such a prime, mined from the AllElectronics value-based catalogue, is

Buys (X, "computer") $\rightarrow$ buys (X, "software") [Support D 1\%, confidence D 50\%], 
$\mathrm{X}$ is a mutable communication to a customer. A certainty, or sureness, of half implies that if a client purchases a PC, there is a half shot that she will purchase.

A $1 \%$ bolster implies that $1 \%$ of the considerable number of exchanges under investigation demonstrate that $\mathrm{PC}$ and programming are bought together. This affiliation lead includes a solitary property or predicate (i.e, purchases) that rehashes. Affiliation decides that contain a solitary predicate are alluded to as single-dimensional affiliation rules.

An evidence mining agenda may determinemembership rules resembling

$\operatorname{Age}(X, " 20.29 ") \wedge \operatorname{revenue}(X, “ 40 \mathrm{~K} .49 \mathrm{~K} ") \rightarrow \operatorname{bargains}(X$, "laptop") [Support 2\%, confidence D 60\%]
The decide demonstrates that of the AllElectronics clients under review, $2 \%$ are 20 to 29 years of age with a pay of $\$ 40,000$ to $\$ 49,000$ and have obtained a tablet (PC) at AllElectronics. There is a $60 \%$ likelihood that a client in this age and salary.

Gathering will buy a portable workstation. Take note of this is an affiliation including more than one property or predicate (i.e., age, salary, and purchases).

Fig.1 refers about Receiving the wording utilized as a part of multidimensional databases, where each credit is alluded to as a measurement, the above administer can be alluded to as a multi-dimensional association lane the demonstration.

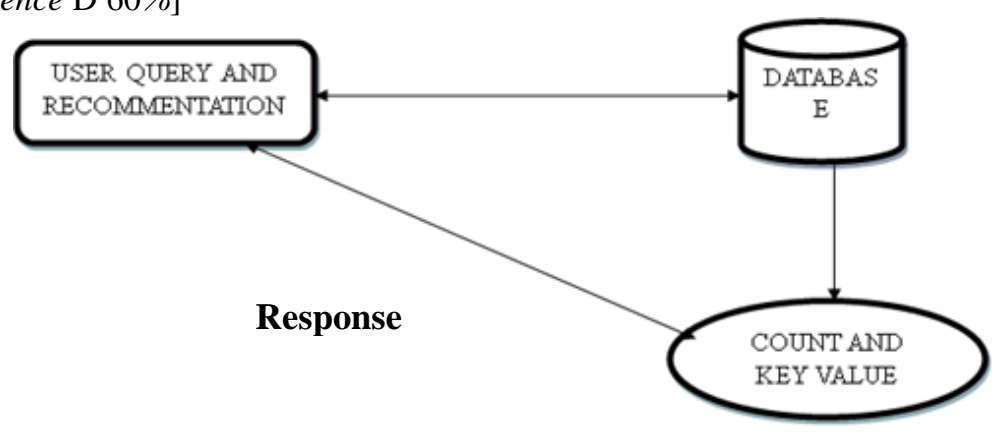

Figure 1. User query approach

\subsection{Producing connotation rubrics from frequent itemsets}

Once the recurrentitemsets from trades in a catalogue $D$ have been found, it is Conventional advancing to generate strong connotation rules from them where strong connotation rules mollify both slightest support and minutestpoise.

Confidence $(A \rightarrow B)=P(B \mid A)=$ support count $(A$ $\mathrm{U} B) /$ support count $(A)$

The conditional probability is expressed in terms of item set support count, where Sustenance count $A \mathrm{UB}$ is the numeral of industries containing the item sets $A \mathrm{U} B$, and Provision reckoning $(A)$ is the figure of dealing the article set $A$.

\subsection{FP growth}

Fig.2 demonstrates the working procedure of FP growth. Visit design development, or just FP-development, which embraces a partition and-vanquish methodology as takes after. To begin with, it packs the database speaking to successive things into a regular example tree, or FP-tree as shown in figure 2 , which holds the thing set affiliation data. It then partitions the packed database into an arrangement of restrictive databases (an uncommon sort of anticipated database), each related with one successive thing or "example section," and mines every database independently. For each "example piece," just its related informational collections should be inspected.

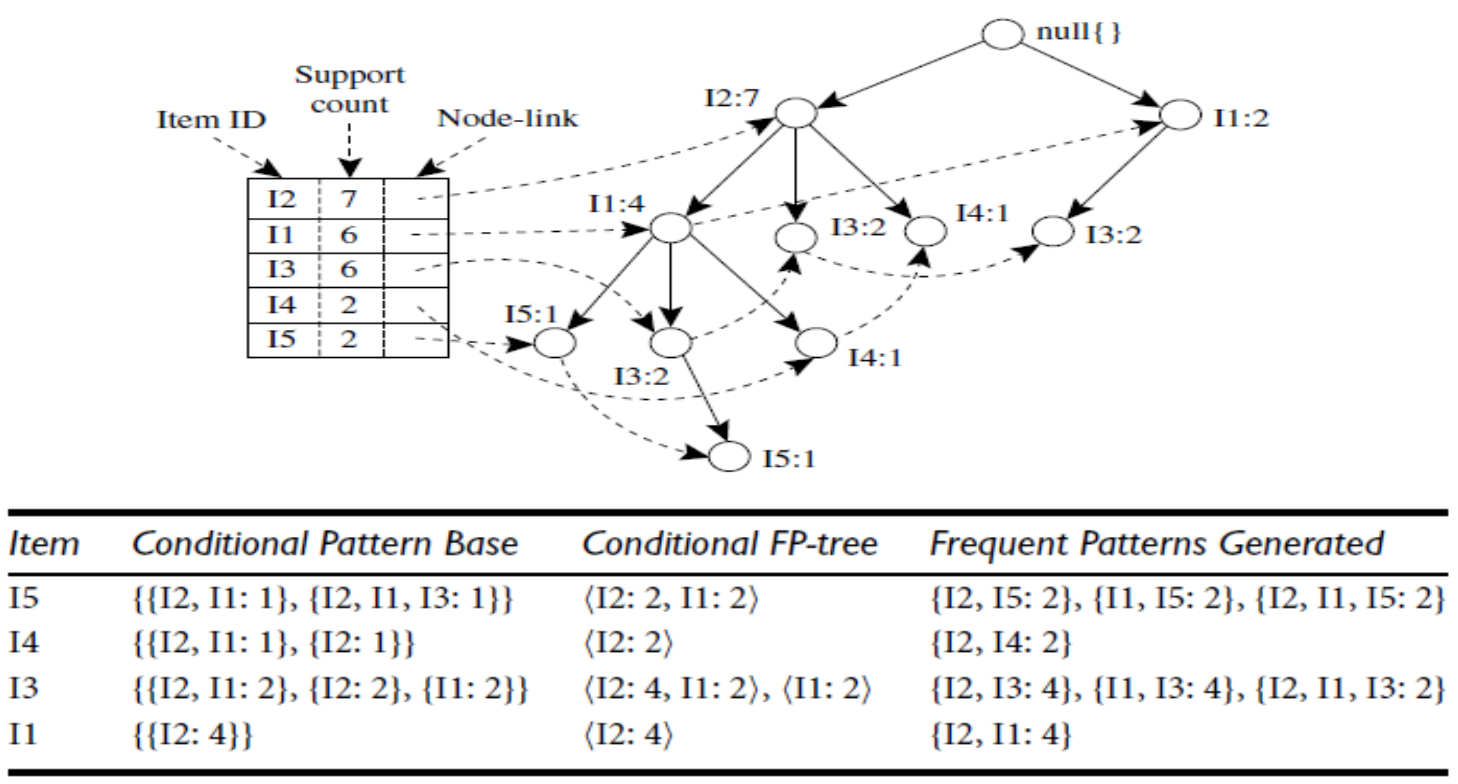

Figure 2. FP-growth tree construction 


\subsection{Tree construction}

In this module the tree has been made for the information to get handled. The info needs to give with the end goal that can recover the substance by framing the tree based structure. The words are organized in tree configuration and it has been recovered by having weightage of diagram. This module concentrates on how information has been embedded into the framework and how the information has been put away into the framework. One the information is required how it has been recovered and how it will get spoke to. Here keeping in mind the end goal to get the information will frame a tree structure of information. Once the information has been put away as tree, while the client ask for the information it will get recovered once they represent the inquiry. Once the tree structure has been framed in view of the substance it will be simple for the information recovery. Just thusly we can recover the regular thing set mining.

\subsection{TM algorithm}

Utilizing TM calculation the data sets which has been gathered that will go under the examinations for finding the weightage of the chart which is actualized keeping in mind the end goal to get the continuous words that has been utilized. The entire datasets have been gathered here and thus it produces weightage of words.

\subsection{Mining top-k high utility itemsets}

A promising arrangement is to rethink the undertaking of mining HUIs as mining top-k high utility thing sets. The thought is to accurately control the yield measure and find the thing sets with the most noteworthy utilities without setting the edges, let the clients indicate $\mathrm{k}$, i.e., the quantity of craved thing sets, rather than saying the base utility limit. Setting $\mathrm{k}$ is more instinctive than setting the limit since $\mathrm{k}$ speaks to the quantity of thing sets that the clients need to discover though picking the edge depends basically on database qualities, which are regularly obscure to clients. The past test is the means by which to successfully expand the min_util Border confine without missing any top-k HUIs.

A decent calculation is one that can viably build the cutoff amid the mining procedure. Notwithstanding, if an off base strategy for expanding the point of confinement is utilized, it might bring about some top-k HUIs being pruned. Consequently, how to raise the point of confinement productively and viably without losing any top-k HUI is a pivotal test for this work. In this paper, the greater part of the above difficulties by proposing a novel system for top-k high utility thing set mining, where $\mathrm{k}$ is the coveted number of HUIs to be mined is locations.

\section{PERFORMANCE ANALYSIS}

FP growth* is the speediest among every one of the calculations with which is tested. The correlation, in any case, is uncalled for. For instance, FP-tree development ought to be slower than the exchange tree development, in any case, in FPgrowth*, the execution of FP-tree development is speedier than our usage for exchange tree development. On account of a base support of 0.5 percent, FP-growth keeps running in $1.187 \mathrm{~s}$, while the development of exchange tree alone in the TM calculation takes $1.281 \mathrm{~s}$. The runtime contrast between FP-development and FP-growth* is not all that extensive in the paper of FP-growth* as in this analysis utilizes an alternate usage of FP-development), which shows that the execution assumes an extraordinary part.

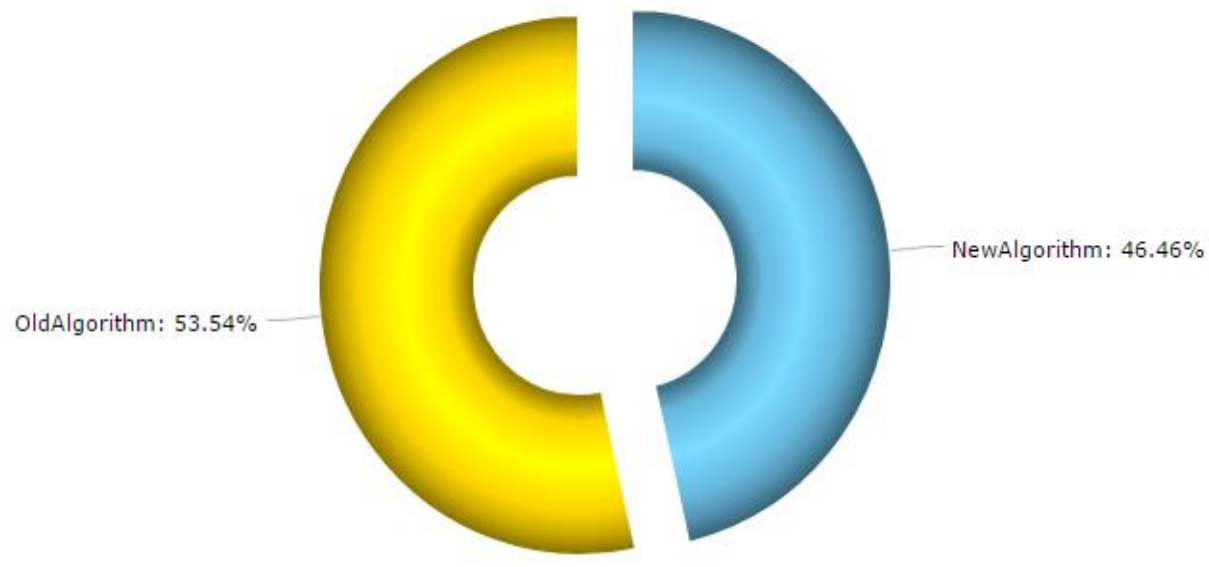

Figure 3. FP growth and TM algorithm performance - data wise difference

Fig. 3 demonstrates the working procedure of old and new algorithm difference in performance and data wise mainly using TM algorithms by tree creation in order to mine top-k high utility item sets.

\section{CONCLUSION}

In this paper, we have proposed another approach, TM, utilizing the vertical database portrayal. Exchange ids of each item set are changed and compacted to nonstop exchange interim records in an alternate space utilizing the exchange tree and regular item sets are found by exchange interims crossing point along a lexicographic tree top to bottom first request. This pressure significantly spares the crossing point time. Through tests, the TM calculation has been appeared to increase critical execution change over FP-development and dEclat on informational collections with short successive examples and furthermore some change on informational indexes with long incessant examples. We have likewise 
played out the pressure and time examination of exchange mapping utilizing the exchange tree and demonstrated that exchange mapping can incredibly pack the exchange ids into consistent exchange interims, particularly when the base support is high. In spite of the fact that FP-growth is speedier than TM in this examination, the correlation is out of line. In our future work, we planned to enhance the implementation of the TM calculation and make a reasonable examination with FP-growth.

\section{REFERENCES}

[1] Han J, Pei J, Yin Y. (2000). Mining frequent patterns without candidate generation. Proc. ACM SIGMOD Int'1 Conf. Management of Data, pp. 1-12.

[2] Bonchi F, Giannotti F, Mazzanti A, Pedreschi D. (2005). ExAnte: A preprocessing method for frequent-pattern mining. Intelligent System 20(3): 25-31. https://doi.org/10.1109/MIS.2005.45

[3] Bonchi F, Goethals B. (2004). FP-Bonsai: The art of growing and pruning small FP-trees. Proc. 8th PacificAsia Conf. Adv. Knowl. Discovery Data Mining, pp. 155-160.

[4] Agrawal R, Srikant R. (1994). Fast algorithms for mining association rules. Proc. 20th Int'l Conf. Very Large Data Bases (VLDB), pp. 487-499.
[5] Pei J, Han J, Mortazavi-Asl B, Wang JY, Pinto H, Chen QM, Dayal U, Hsu MC. (2004). Mining sequential patterns by pattern-growth: The prefix span approach. Knowledge and Data Eng 16(10): 424-1440. https://doi.org/10.1109/TKDE.2004.77

[6] Li PX, Chen JP, Bian FL. (2004). A developed algorithm of a priori based on association analysis. Geospatial Information Science 7(2): 108-112.

[7] Wang JY, Han JW, Lu Y, Tzvetkov P. (2005). TFP: An efficient algorithm for mining top-k frequent closed itemsets. Transactions on Knowledge and Data Engineering 17(5). https://doi.org/10.1109/tkde.2005.81

[8] Tseng VS. et al. (2013). Efficient algorithms for mining high utility item sets from transactional databases. Knowledge and Data Engineering 25(8).

[9] Cagliero L, Garza P. (2014). Infrequent weighted item set mining using frequent pattern growth. Knowledge and Data Engineering 26(4).

[10] Liu JQ, Wang K, Fung BCM. (2016). Mining high utility patterns in one phase without generating candidates. Knowledge and Data Engineering 28(5): 1245-1257. https://doi.org/10.1109/TKDE.2015.2510012

[11] Janarthanan P, Rajkumar N, Padmanaban G, Yamini S. (2014). Performance analysis on graph based information retrieval approaches. AMSE Journal -Series: Advances-D 19(1): 1-14. 